\title{
Coulisses
}

Revue de théâtre

12 | Printemps 1995

Varia

\section{Les acteurs de Peter Brook}

Ouriel Zohar

\section{(2) OpenEdition}

Journals

Édition électronique

URL : http://journals.openedition.org/coulisses/3818

DOI : $10.4000 /$ coulisses.3818

ISSN : 2546-9460

\section{Éditeur}

Presses universitaires de Franche-Comté

\section{Édition imprimée}

Date de publication : 1 mai 1995

Pagination : 40-50

ISSN : 1150-594X

\section{Référence électronique}

Ouriel Zohar, «Les acteurs de Peter Brook », Coulisses [En ligne], 12 | Printemps 1995, mis en ligne le 15 mars 2019, consulté le 21 octobre 2019. URL : http://journals.openedition.org/coulisses/3818 ; DOI : 10.4000/coulisses.3818

Ce document a été généré automatiquement le 21 octobre 2019

Coulisses 


\title{
Les acteurs de Peter Brook
}

\author{
Ouriel Zohar
}

Lorsqu'en 1970, Peter Brook pénétra pour la première fois avec Micheline ROZAN Directrice de son Théâtre, dans la salle des Bouffes du Nord à Paris, il dit immédiatement: «il sera un jour nécessaire que ces murs chantent $»$. Depuis naturellement, la musique a pris une part considérable dans son travail théâtral, jusqu'à ce qu'un jour il décide de mettre en scène un opéra. Bien évidemment, il se heurtera aux limites dues à l'architecture même de la salle et de la scène car il n'y a pas de place pour un grand orchestre ni la participation de nombreux figurants. Malgré cela, l'orchestre peut se déployer harmonieusement dans le cadre architectural existant, surtout depuis que le théâtre a reçu l'aide financière du nouveau Directeur du Théâtre de l'Opéra de PARIS. Cette aide a permis de monter l'opéra de CARMEN réalisé par BROOK en 1981. Ce spectacle a fait le tour du monde et s'est rendu notamment en Israël en 1987.

2 L'idée de вRоOK de monter CARMEN, s'inscrit dans la continuité de son travail avec sa troupe internationale. Depuis longtemps déjà, il songeait à réadapter et revivifier un opéra dans des conditions différentes de celles qu'il avait rencontré auparavant. Il a trouvé son inspiration tout d'abord dans l'adaptation de J.-Claude CARRIERE mais aussi dans son désir de créer de nouvelles relations entre les chanteurs et le public, entre ses acteurs et les chanteurs. L'agencement et l'architecture des théâtres classiques dans lesquels les opéras étaient montés auparavant étant totalement contraires aux goûts et conceptions de Peter вRоок. Dans ce style de théâtre traditionnel, la scène était grande, 
envahie d'instruments techniques et électroniques, l'orchestre était imposant, les salles étaient immenses. Cet ensemble de faits constituaient le genre de relations théâtrales que nous connaissons tous.

BROOK, essaiera de créer plus de concentration, de vérité et d'intimité.

4 Pour permettre une expression théâtrale plus directe et proche du public, la structure même du Théâtre des Bouffes du Nord paraissait idéale. En effet, la distance entre la scène et le dernier rang de la salle ne dépasse pas plus de dix mètres.

Les acteurs de son théâtre ont reçu une préparation des années durant totalement différente de celle des chanteurs. Aussi, pendant longtemps, le travail des chanteurs et des acteurs ne donna pas de fruits immédiats. Seulement après un an de collaboration, les chanteurs commencèrent à comprendre progressivement les intentions de BROOK et ses méthodes de travail.

Il monta d'abord les trois premières versions de CARMEN avec alternativement pour rôle principal soit une Carmen française, une Carmen autrichienne et une Carmen israélienne, pour finalement doubler le nombre des actrices et arriver à six interprètes dont trois américaines. Il réalisa ainsi l'idée à laquelle il tenait beaucoup et que pour chaque spectacle il fallait jouer différemment, car la vie change aussi continuellement. Il réalisa cette œuvre avec six équipes différentes.

7 L'interview réalisée avec d'anciens acteurs de P. BROOK: Alain MARATRA et Jean-Paul DENIZON a eu lieu après les répétitions de l'après-midi et avant la représentation du soir de l'opéra CARMEN. Nous avons parlé un peu de la répétition, mais comme BROOK tient à garder le secret sur son travail, il est parfois difficile de leur faire aborder ce qui vient de se passer durant les répétitions quelques instants auparavant.

0. zoнar : Comment avez-vous rencontré Peter вRоок?

A. MARATRA : Moi, j'ai d'abord fait des études.

O. ZOHAR : Ici à Paris?

A. MARATRA : Durant trois années j'ai étudié le théâtre à Bruxelles et après je suis venu jouer à Paris dans un théâtre de banlieue. Jean-Pierre vinCENT qui est metteur en scène a vu le spectacle. A cette époque-là, Peter BROOK recherchait des acteurs pour TIMON D'ATHENES, il y a 7 ans de cela ${ }^{1}$. Jean-Pierre VINCENT après m'avoir vu a parlé à Peter qu'il connaissait et un beau jour j'ai reçu un télégramme m'invitant à me présenter. Je suis venu le voir, nous avons discuté et nous avons essayé pour TIMON D'ATHENES.

O. zoнar : Si je m'en tiens au propos de la chanteuse israélienne Zehava gaL, je comprends que vous ne vouliez plus travailler qu'avec Peter вrook, vous voulez rester avec lui.

A. MARATRA : Ce n'est pas que je ne peux plus travailler ailleurs, mais c'est surtout important pour moi maintenant de rester avec Peter parce qu'il a un projet qui m'intéresse beaucoup : LE MAHABARATA. Tout ce que j'ai pu faire jusqu'à présent n'était qu'une préparation pour ce spectacle et je suis d'autant plus motivé que la réalisation de ce projet approche. Cela demande un tel effort de concentration, de recherche sur la voix, la pratique d'exercices en tout genre qu'il n'y a pas de temps pour faire autre chose. C'est une période comme ça qui me convient très bien. Comme CARMEN en ce moment par exemple, je ne vais pas partir maintenant alors que je sens tellement bien le rôle. On m'a fait d'autres propositions ailleurs et j'ai refusé car 
je me sens bien ici et maintenant. Il y a beaucoup de travail, nous commençons les répétitions à 10 heures du matin et nous terminons le travail très tard la nuit.

O. zoнAR: Le travail est-il différent avec CARMEN et les nouveaux chanteurs qui ne connaissaient pas les méthodes de вRоoк et votre groupe international déjà sensibilisé ? Dans la pièce la CONFÉRENCE DES oISEAUX par exemple, vous avez travaillé durant des années de répétitions, de préparations à New-York, à Paris, en Afrique. Les rencontres avec de nombreuses communautés, de peuples différents et les réactions d'un public universel vous ont aidé à construire cette magnifique et superbe pièce. Même après, durant les représentations vous avez continué à faire des corrections. II y a dans ce travail quelque chose de très étonnant. Vous êtes un groupe spécial et original.

A. MARATRA : Oui, notre travail est spécial. Nous essayons d'élaborer quelque chose de tout à fait nouveau et l'activité ne se termine jamais avec la représentation, il y a une recherche continuelle même durant les spectacles. On retrouve dans toutes les représentations de $\mathrm{P}$. BROOK la même chose : la recherche de la vie au théâtre ! Mais si l'on prend par exemple: Mesure pour Mesure ; La Cerisaie ou Ubu, rien n'est jamais pareil. La recherche de BROOK consiste à faire vivre les mots. Il n'y a pas de règle non plus, parce que nous ne pouvons jamais dire que nous avons trouvé ce que je pourrais appeler une "synthèse d'énergie "! Ainsi par exemple, nous avons joué Ubu avec une énergie très physique tandis que dans la Cerisaie qui est classique, nous avons élaboré un travail plus intériorisé. Malgré cela les deux pièces demandent la même énergie, la même vérité, la même vie. Une vie qui doit transparaître et sortir de là, c'est cela la recherche propre à Peter BROOK je crois?

O. zoHaR : Que veut dire pour vous : «travailler sur la vie dans le théâtre »?

A. MARATRA : On ne travaille pas sur la vie, jamais. On essaye d'enlever des choses inutiles, ou de les remettre, bouger autour, les casser. Par exemple dans PEER GYNT de Henrik IBSEN, à la fin il y a un moment où il épluche un oignon et dit : "...voilà l'homme c'est ça, c'est comme moi, il enlève une peau et encore une peau... " au fond il y a le noyau, il y a le centre. C'est un peu cela, enlever à chaque fois des choses superflues ou en rajouter lorsque l'on est incertain et c'est tout, alors la vie arrive à ce moment précis. La vie en elle-même est déjà tellement compliquée. C'est difficile de rencontrer un autre être et d'avoir un vrai contact de personne à personne. Parfois on s'aperçoit que l'on a été touché par quelqu'un, quelque chose a été échangé, c'est plus que des mots et des idées, mais il est très rare qu'une rencontre de ce type ait lieu. Il nous arrive par exemple de tomber amoureux, comme ça d'un seul coup... ou bien encore quelqu'un vous casse un tabouret sur la tête et vous vous retournez, alors là il y a un véritable échange. Dans la vie, on croise des tas de gens avec lesquels il n'y a jamais un véritable échange, il ne se passe pas grand-chose d'intéressant qui arrête votre course. Il peut s'agir d'une fille qui passe, les cuisses presque complètement à l'air et alors là il se passe quelque chose d'intéressant qui vous alerte, vous réveille. Il faut des choses très fortes et dans le théâtre c'est encore dix fois plus compliqué parce que nous avons de mauvaises habitudes. Je vous l'ai dit pour trouver la vie dans le théâtre ce n'est pas simple. Nous acteurs, il nous faut rejouer Carmen chaque soir et retrouver cette vie, celle qui était là aux répétitions, il y a un an... c'est un combat terrible qu'il faut mener avec nous-mêmes pour retrouver cette inspiration chaque soir. 
O. zoнar : Vous recommencez ce combat avec vous-même ou avec le public ?

A. MARATRA : Avec les deux, toutefois ce combat s'exerce surtout avec nous-mêmes. Néanmoins, nous n'écoutons jamais assez le public car c'est le public qui nous écoute et ceci à différents niveaux. Si nous étions plus près du public nous n'aurions jamais besoin de ce combat. Par exemple, nous avons joué dans les prisons et nous n'avons pas eu besoin de combattre, c'était beaucoup mieux parce que le dragon était là en arrière fond. Le public était là, présent, complètement demandeur d'actions. Nous avons joué devant des prisonniers et ils avaient envie de voir «la vie», de voir quelque chose d'intéressant. Du théâtre, ils s'en fichaient complètement quelque part. Aussi cette expérience nous a beaucoup appris, elle nous a donné un coup de fouet immédiat.

\section{O. zoнar : C'était dans quelle prison?}

A. MARATRA : Fleury Mérogis dans la banlieue parisienne! Là c'est caractéristique, la vie vient tout de suite. Parfois aussi, nous sommes très en forme et dans Carmen par exemple on attaque très fort. Tous sont là, présents, quelque chose se passe et ça va... Hier soir aussi nous avons joué, il y avait beaucoup de monde dans la salle, c'était plein à craquer ce qui nous influence beaucoup et nous aide. Ce phénomène ne vient pas de l'extérieur mais il demande aussi de nous-mêmes. Parfois nous sommes fatigués, cela ne va pas et rien ne passe.

O. zoнar : C'est étonnant ce choix de Peter Brook de monter CARMEN. II n'avait pas monté d'opéra depuis longtemps et je crois-que c'est le premier qu'il monte au Théâtre des Bouffes du Nord?

A. MARATRA : Oui c'est la première fois.

O. zoHAR : Donc ce travail n'était pas très nouveau pour lui ?

A. MARATRA : Totalement, mais au risque de me répéter ses spectacles ne sont jamais identiques. Dans la Cerisaie où je ne jouais pas, là encore le spectacle a été travaillé d'une manière tout à fait différente. Peter a choisi d'autres acteurs que sa troupe habituelle et pourtant à la fin on perçoit le même esprit. Lorsque je suis venu assister à la première de la Cerisaie, je pensais que j'allais détester. Et puis non, on reconnaît la même empreinte : une vie, une vérité, des gens qui jouent et c'est magnifique.

0. zoнar : Ainsi vous dites que chaque soir il y a autre chose! Si je vous comprends bien, la particularité de Peter ввоок c'est la "vérité » qu'il recherche permettant aux acteurs de « revivre » sur la scène et de créer quelque chose de nouveau chaque soir.

J.-P. DENIzon : Oui, lorsque tous le veulent bien !

O. zohar : Et vous, Jean-Paul denizon comment avez-vous rencontré P. BRook?

J.-P. DENIzon : Moi je jouais dans un café théâtre qui s'appelle ou plutôt qui s'appelait car il a disparu depuis : « le coupe-chou ». C'était à Beaubourg. Je jouais une pièce de VERCORS : Le silence de la mer. Un soir, BROOK est venu pour assister au spectacle. Je ne sais pas pourquoi, mais il est venu. Il m'a appelé ensuite. J'ai travaillé avec lui dans la Cerisaie, maintenant dans Carmen et puis l'an prochain le Mahâbbârâtâ.

0. zонав : Quelle différence faites-vous entre le travail du café-théâtre et P. вRоок?

J.-P. DENIzon : Ce n'est pas une question de lieu et cela n'en a jamais été. Avant de rencontrer BROOK, j'ai beaucoup travaillé dans le théâtre et j'ai remarqué qu'il s'agissait toujours d'une question de personne. Tout dépend des gens avec lesquels nous travaillons. La preuve c'est qu'avec Peter on se sent très bien. On peut jouer 
n'importe où avec lui, que ce soit ici aux Bouffes du Nord ou dans une prison, c'est pareil on retrouve la même joie. L'expérience est différente, le lieu modifie l'expérience mais disons que le fond n'est pas modifié.

O. zoHaR : En quoi l'expérience avec P. Brook diffère-t-elle selon vous ?

J.-P. Denizon: Tout dépend de la personne avec qui l'on travaille. Lorsque je travaillais au café-théâtre c'était avec un autre metteur en scène plus directif. Il laissait moins de liberté aux comédiens, puisqu'il se faisait une idée a priori sur le personnage. Ainsi « son » personnage, c'est-à-dire le personnage que j'interprétais ne pouvait se jouer que d'une certaine manière qui était la sienne. Or, avec Peter BRooK ce qui est fondamentalement différent, c'est que même s'il a lui-même une conception très personnelle sur les rôles qu'il va nous faire interpréter, il laisse toujours la liberté d'expression à l'acteur qui se trouve en face de lui. Il n'a pas d'à priori. L'artiste peut continuellement s'exprimer au maximum de ses potentialités et enrichir énormément son personnage.

O. zонав: Vous dites que le travail se fait toujours en collaboration mais est-ce que Peter вRоок apporte des détails précis pour l'interprétation des personnages?

J.-P. DENIzon: Il n'y a pas de règle. Peter travaille beaucoup en fonction de la nécessité. S'il ressent qu'à tel endroit telle chose est utile, il nous dira de le faire, mais il nous expliquera pourquoi cela lui paraît important et si l'on en comprend le bienfondé alors on le fait. Parfois il ne dit rien tout au long d'une scène et c'est nous qui la trouvons entièrement. Un autre jour au contraire, nous ferons un travail très précis parce qu'il sent qu'il y a quelque chose à trouver ou il perçoit que nous nous trompons, toutefois il n'y a jamais de règles immuables. Nous travaillons tous ensemble sur une réalité que nous essayons de construire. Un acteur aura une idée qu'il suggérera, un autre exprimera une idée différente, ou bien encore Peter dira : «Et si l'on faisait cela?». Alors nous essayons, tout est très pragmatique, cela se construit ou ne se construit pas!

0. zонав : C'est aussi un travail d'expérimentation. Je comprends que vous Jean-Paul, vous trouviez votre intérêt dans l'improvisation, c'est ce qui vous enthousiasme et vous inspire, tandis qu'Alain recherche plutôt la vie, la création permanente d'une vie différente en changement?

J.-P. DENIzon : Je ne crois pas que nos motivations soient tellement différentes. Nous n'employons pas les mêmes mots mais nous recherchons les mêmes choses en fait.

A. MARATRA : Ce qui est très intéressant c'est que nos méthodes de travail sont totalement différentes. Nous n'avons pas la même approche du théâtre. De même dans la vie, l'un sera plutôt sportif, l'autre plus tourné vers la vie intellectuelle. Quelqu'un de plus émotionnel à qui l'on demandera : «Tu veux un pain? " (il exagère avec un cri) répondra : «Quoi, qu'est-ce que tu me dis?» (cri). Il y a des gens comme cela. Lorsque l'on joue, il est difficile de parler de son propre jeu. Certains disent que Carmen est un très bon résultat car ВRоок est un excellent technicien et ses acteurs, il faut s'exprimer avec des mots nuancés, nous sommes en fait très différents fondamentalement.

J.-P. DENIzon : C'est normal et c'est très bien. Cette diversité même créera justement la vie que nous recherchons. Pour nous, le plus important c'est d'abord de toucher le public, c'est notre premier objectif. Lui communiquer une expression allant au-delà même des idées contenues dans la pièce interprétée à ce moment précis. A partir du 
moment où les idées sont bien énoncées elles seront comprises sans problème. Toutefois au-delà de cela, il y a ce que nous nous essayons de trouver dans la création et qui doit être absolument «vrai ». Je ne nommerais pas cela forcément la vie, je dirais que c'est quelque chose de "vrai ", qui peut paraître plausible à tout public et qui peut donc toucher. Bref un sentiment humain avant tout. Essayer de communiquer une émotion, de faire passer une chose que l'on ne comprend pas forcément, mais qui nous semble importante. C'est un peu ce que nous faisons avec Carmen actuellement, nous essayons de trouver une espèce de magie et je crois qu'elle peut passer et être ressentie par tous les publics.

0. zoнar : Quelle est cette magie?

J.-P. DENIZON : Nous ne savons pas. Nous l'avons ressentie dans la scène où se forme les trois cercles entourés d'un feu.

0. zohaR : Presque à la fin de Carmen!

J.-P. DENIzon : Oui. Il fallait réussir à manifester de la magie. Nous avons beaucoup cherché en utilisant des techniques variées et puis un jour nous avons trouvé. A quoi cela tient-il nous ne le savons pas. Objectivement cela tient à quoi ? Trois feux, un cercle et un point !

0. zoнar: Pensez-vous que ce que vous avez trouvé dans l'improvisation; ce feu et ce cercle souligné dans le sable sont quelque chose de beau ? Soudainement ce feu véritable sur la scène c'est très original!

J.-P. DENIzon : Oui c'est très joli, je suis d'accord. Mais ce n'est pas suffisant. Notre recherche et celle de Peter n'est jamais uniquement d'ordre esthétique, jamais !

O. ZOHAR : Vraiment?

J.-P. Denizon : S'il n'y avait que la recherche du beau, cela n'aurait aucun intérêt. Il faut qu'une émotion passe, que s'exprime une vérité, une vérité humaine, c'est cela qui compte avant tout! Si c'est esthétique en plus tant mieux.

A. MARATRA : Comment avons-nous trouvé cette scène c'est magnifique ? Nous avons travaillé la scène avec GARCIA et CARMEN. C'était le point de départ : GARCIA-CARMEN. Moi j'ai travaillé en improvisant, c'était à l'arraché, il se foutait d'elle, il rigolait... GARCIA c'est une scène un peu drôle, mais cela n'avait aucune existence et moi j'étais content... Soudain Peter a dit que nous ne trouvions pas l'essence, que nous tournions autour. Pourquoi ? nous ne réussissions pas et puis d'un seul coup Peter a suggéré : «Bon, mettons tout dans le noir, dans la pénombre comme ça pour voir ». Nous avons essayé. Subitement je me suis trouvé dans une situation où j'étais forcé d'y arriver. Comme d'un coup on ne sait pas très bien comment quelqu'un vous étonne, c'est Jean-Paul qui a eu l'idée : « Mais on pourrait faire un feu !».

J.-P. DENIzon : Non, c'est Maurice !

O. zohar : Maurice béNICHOU!

A. MARATRA : Nous avons essayé, nous avons « mis le feu » et voilà !

J.-P. DENIzon : Un jour, alors que je répétais, je travaillais sur la «vieille», j'avais allumé le feu et je n'avais plus rien à faire. Nicole la costumière avait déposé un tube de poudre et j'avais ce tube de poudre dans les mains, alors j'ai fait un cercle autour du feu, comme ça parce qu'il fallait s'occuper! Généralement on pense que les idées sont le résultat d'une longue réflexion très profonde, voire même très pénible, 
quelque chose qui met un temps infini à se concrétiser. Etonnamment, nous pensons aussi que les idées surgissent grâce à notre volonté en quelque sorte, or souvent, or souvent les grandes idées viennent spontanément.

0. zoнar : C'est toutefois le résultat de beaucoup de travail !

J.-P. DENIZON : Evidemment.

A. MARAtra : Ce qui est extraordinaire c'est qu'il ne s'agit pas d'un hasard. C'est absolument le contraire du hasard et c'est toujours ainsi, parce que l'improvisation, la vie est ici. On est là, on attend, on est sensible à ce qui se passe. Jean-Paul Denizon a expliqué « la trouvaille » de la poudre et du feu qui est tombé exactement au moment où il le fallait. Il a expliqué cela comme un acteur et non pas comme un penseur. Ainsi, tout au long du processus de recherche de l'acteur, à un moment donné quelque chose arrive ou tombe. Alors on se dit : «tiens, maintenant je vais le faire! » mais pour tout cela, il faut les conditions !

J.-P. DENIzon : Notre travail, c'est d'être toujours, toujours à l'affût de la vérité que nous cherchons à exprimer. Donc, le moindre indice qui surgit, qui apparaît sur la scène nous l'attrapons et nous nous en servons, on expérimente en quelque sorte.

A. MARATRA : J'ai fait mes études de théâtre en Belgique vous le savez. Il y a une chose au théâtre français que je n'ai jamais bien comprise et qui heureusement n'existe pas chez P. BRоок. Au début du travail les Français lisent ce qu'ils appellent «neutre » ce qui à mon sens est une erreur monumentale. Avec Peter, jamais nous n'avons répété quelque chose techniquement, mécaniquement. Même à la première lecture, tout de suite on cherche. "Ce n'est pas vivant» - Il faut immédiatement se mettre en position de recherche, de faire vivre le texte. Quand la pièce est proposée aux acteurs, au moment des exercices, dès le début du travail, il ne faut pas dire : «Je le ferai bien dans trois jours ». Avec Peter, il y a l'improvisation continuellement mais avec les chanteurs un peu moins car ils improvisent très peu. C'est ce que je trouve intéressant : improviser sur scène.

O. zoHaR : Les chanteurs ont beaucoup de difficultés à improviser, parce qu'ils viennent d'un autre univers.

A. MARATRA : Pas tous. Au début c'était très difficile, c'était terrible et même presque impossible, personne n'était capable d'improviser.

J.-P. DENIzon: Le problème dans l'interprétation du chant, c'est que toute la concentration reste seulement au niveau de la gorge, dans la composition de telle ou telle note, la donner plus ou moins dramatiquement ou plus légèrement mais cela reste intellectuel. Le corps des chanteurs ne bouge jamais, ou presque jamais. Aussi on ne leur apprend pas, parce que ce n'est pas une préoccupation pour eux, car il y a longtemps que l'opéra s'est séparé du jeu et l'opéra maintenant est devenu un lieu où l'on fait des prouesses techniques uniquement. Ici, nous essayons toujours de combattre au maximum les techniques dans la mesure où elles sont limitatives de l'expression, qu'elles enferment l'artiste et son expressivité dans le cadre trop précis et étroit. Or, nous les acteurs, nous avons besoin continuellement de rendre la vie et la vie ne peut se trouver dans une technique, même s'il existe des techniques pour bien vivre. La vie est immense et une technique ne peut rendre compte de toute une vie, de toute la vie tout au moins, donc il faut apprendre cela aussi. Il y a eu un 
combat très dur pour les chanteurs qui doivent respecter des nécessités techniques absolues que l'on ne peut briser.

0. zoнar : D'après ce que je comprends certains ont compris, d'autres non?

J.-P. DENizon : Cela dépend toujours des personnes avec qui l'on travaille. Certains essaient de créer et cela leur plaît énormément, ils en ont envie. D'autres, malgré le plaisir qu'ils peuvent y prendre n'y arrivent pas. Enfin, il y a aussi ceux qui n'ont pas forcément envie de travailler comme cela. Si l'on prend au hasard des gens réunis dans un café, il y aura 100 personnes différentes et c'est cela la vie.

O. zонAR : Et maintenant la préparation du MAнаввARATA. C'est une pièce indienne avec des représentants de l'Inde venus ici spécialement.

A. MARATRA : Cela n'a rien à voir avec la pièce, c'est le travail préparatoire.

O. zoнar: J'ai été étonné au début de notre entretien, lorsque vous avez dit que tout le travail effectué jusqu'à présent était une préparation pour le MAHABBARATA.

A. MARATRA : Absolument, je sens cela très puissamment. C'est la première fois que je perçois vraiment ce que veut dire jouer. Si quelqu'un me demande : comment as-tu joué le rôle? Je répondrais avec emphase que j'ai mis sept ans pour pouvoir dire cela et jouer ce rôle-là. Il a fallu bien des étapes, des expériences assez terribles avec les pièces de Peter, pour que par hasard je sois là et j'arrive à jouer Carmen. Oui, j'étais là par hasard. Nous avons employé des méthodes spéciales et moi le comédien j'y ai trouvé quelque chose de primordial.

J.-P. DENIzon : Le travail pour un comédien est toujours une préparation pour le futur, je crois. Nous essayons de progresser continuellement du moins je l'espère. Puisque l'on apprend toujours quelque chose de nouveau, la représentation suivante doit automatiquement être meilleure. Cela fait aussi sept ans que BROOK pense à cette pièce du MAHABBARATA. Chaque fois qu'il montait un nouveau spectacle il avait derrière la tête ce MAHABBARATA là !

O. zoHar : Vous jouerez aussi dans la pièce ? Vous connaissez déjà votre rôle?

J.-P. Denizon : Non. Il y a une foule de rôles, il y a toute l'Inde... alors cela je ne le sais pas encore.

O. zoHaR : Alors sur quoi travaillez-vous?

J.-P. DENIzon: Nous cherchons actuellement les moyens. Nous nous efforçons de trouver comment communiquer à un public occidental une culture qu'il n'a pas encore approchée, tout à fait opposée à la culture occidentale. Quels sont les moyens de communication, quelles sont les barrières qui se présentent à nous et comment les faire tomber. Comment passer ces barrières pour pouvoir donner au public quelque chose qu'il ne ressentait pas auparavant, qui ne ressort pas de sa culture et lui apparaisse néanmoins très vraie, assez normale et très naturelle. C'est un travail énorme. Il est évident qu'il n'est pas facile d'expliquer tout le Mahabbarata en quelques heures.

O. zoHaR : Combien d'heures?

J.-P. DENIZON : Nous ne savons pas exactement.

A. MARATRA : Il y a 18 volumes... Il y a 18000 vers. 


\section{0. zoHAR : Je crois que Peter a parlé de 200000 vers?}

J.-P. DENIZON : Le MAHABBARATA qui pourtant fait partie de la culture indienne, est joué en 18 soirées de 4 heures ce qui est énorme! Nous nous attachons tout d'abord à condenser ces textes car nous ne pourrons pas tout jouer.

O. zohar : Jean-Claude carriere travaille à cette mise en forme.

A. MARATRA : Oui et ce qui est intéressant c'est que nous rencontrons les mêmes problèmes qu'avec l'Ancien Testament. Si l'on essayait de monter une scène avec MoÏsE lorsque Dieu lui apparaît sous la forme d'un feu, nous aurions l'impression qu'il s'enflamme.

O. ZoHaR : Comme une voix.

A. MARATRA : Le problème serait alors de jouer Moïse à ce moment-là et c'est la grande difficulté que nous rencontrons avec le MAHABBARATA. J'ai vu de nombreuses tentatives cinématographiques aux Etats-Unis par exemple, mais Moïse n'est pas un homme ordinaire. Il a la puissance que toutes les nations connaissent. Moïse a parlé avec Dieu et il a fait descendre les Tables de la Loi. Essayez d'interpréter Jésus lorsqu'il a marché sur l'eau. Il a une certaine force. Il avait une énergie formidable. C'est comme le personnage de Siegfried et de tous les héros. Il ne suffit pas de leur faire porter une barbe avec une robe blanche car le public ne marchera jamais. La plupart du temps les types qui s'en affublent sont incapables de jouer un tel rôle, tout simplement ils ne peuvent pas porter cela. Il faut voir les yeux de ces types et il faut voir les yeux des Saints dans la Bible. C'est très difficile à exprimer. Par exemple comment faire parler Moïse, comment lui rendre la parole au point qu'on puisse le sentir, l'écouter.

J.-P. DENIzoN : Comment rendre crédible à notre époque Moïse et le buisson ardent? C'est un vrai problème au théâtre. Cet arbre qui reste enflammé sans cesser de brûler. Comment va-t-on faire croire cela aux occidentaux ? Cela peut faire sourire. C'est tout à fait différent d'une lecture qui peut toujours paraître crédible parce qu'elle reste dans l'imaginaire. Chacun peut se la représenter à sa manière et pas forcément avec des images.

0. zонав : Voulez-vous dire que vous rencontrez des problèmes de décor?

J.-P. DENIzon : Tous les problèmes se posent à nous. Il y a trois jours par exemple nous avons butté sur un texte avec Peter car dans la traduction on parlait de tous les peuples qui se rassemblent pour faire la guerre. Il y avait une énumération : «Les BANGAS, les DVORAS, les CARACS » ... donc des noms indiens énumérés et d'un seul coup « des montagnes»! Ce mot français placé à côté des autres devenait impossible à prononcer car il sonnait faux à l'oreille.

0. zoнar : Peter ввоок est venu d'Angleterre déjà avec cette idée. II pensait peut-être que SHAKESPEARE serait plus tangible pour le public si on le traduisait d'une manière plus quotidienne, plus directe, plus abordable. En Angleterre on le joue dans un style tellement traditionnel que plus personne ne le comprend. Aussi l'anglais de SHAKESPEARE paraît souvent trop archaïque. Si l'on prenait la traduction de la Bible de la même manière, concrète et tangible comme l'entend P. ввоок cela pourrait être une solution.

A. MARATRA: Il y a les problèmes de traduction mais il $y$ a aussi ceux de l'interprétation! 
O. zоHAR : La traduction est déjà une interprétation.

A. MARATRA : Oui mais il faudra aussi jouer des demi-dieux. Il y aura des batailles qui durent longtemps. Si l'on veut tuer un éléphant par exemple est-ce par la ruse ou par la force? Essayer de combattre un éléphant par la force, cela veut dire que le comédien doit être physiquement suffisamment fort pour tuer un éléphant. Par moment, il y a BHIMA...

J.-P. DENIzon : Bhima c'est l'homme le plus fort du monde.

A. MARATRA : Dans l'histoire il est dit que chaque fois que BHIMA veut passer quelque part et qu'il rencontre un obstacle, une montagne par exemple, il la soulève et la déplace. Il y a aussi SHIVA en haut, qui prend la montagne, la met de côté et passe. C'est écrit et c'est crédible comme dans un conte. Alors maintenant, imaginez un acteur aux Bouffes du Nord à qui l'on dit: « vas-y voyage avec les cinq frères et déplace la montagne »...

O. zohar: Dans la conférence des oiseaux aussi, il y a beaucoup d'événements qui se passent dans les montagnes, dans les ravins.

A. MARATRA : Nous avons fait un énorme travail et c'est possible. Mais il s'agissait d'une histoire condensée.

J.-P. DENIzON : Et puis dans La Conférence des oiseaux vous n'avez vu que le résultat, c'est-à-dire la pièce.

O. zoнar : Malheureusement.

J.-P. DENIzON : Il aurait fallu voir le processus de réalisation afin que le travail effectué paraisse crédible au spectateur.

O. zohar : Ce qui est passionnant chez Peter avec LA Conférence des oiseaux par exemple, c'est d'essayer de deviner tout le travail qui se dessine derrière. Nous retrouvons toujours cela chez Peter et dans tous ses spectacles. Avec LA CERISAIE; MEsUre POUR MEsURE et en ce moment la tragédie de CARMEN, on éprouve les mêmes sentiments. Nous nous posons les mêmes questions, que s'est-il passé hier ici, ou avant-hier avant la représentation?

A. MARATRA : Il s'agit d'un « truc secret » dont il ne faut pas trop parler. Par « secret » j'entends cet échange dont vous parlez et que vous avez ressenti. Il arrive que l'on parle dans les débats après les spectacles, mais en fait on casse certaines choses dans les conceptions du public. On ne peut pas toujours répondre aux gens lorsqu'ils nous posent des questions après le spectacle : «comment avez-vous joué cela et pourquoi avez-vous joué comme cela ? » Lorsque l'on joue il se produit vraiment un échange. Il y a une circulation perpétuelle entre l'acteur et le public. Ce regard, est la chose la plus merveilleuse qui soit dans le théâtre. Nous sommes tellement près qu'il s'agit presque d'une conversation inconsciente tout le temps que dure le spectacle. Les gens ferment les yeux et c'est magnifique. Il n'y a qu'au théâtre qu'il soit possible de jouer et regarder en même temps le public. Nous les acteurs, nous ressentons très bien lorsque nous ne jouons pas juste, " on fait du théâtre », on triche! Alors l'autre, celui qui regarde voit une espèce de " $\mathrm{mec}$ " qui se cache derrière un personnage. Il voit cela d'un seul coup et c'est plein de charme et puis le public croit et il est là, ça l'amuse. En même temps il croit et il ne croit pas. Il sent le type qui s'amuse, c'est cela le plus important. 
О. ZонаR : Vous n'aimez pas parler du travail « laboratoire»?

A. MARATRA: J'ai toujours un peu peur de parler. Cette chose qui s'échange est impalpable. Lorsqu'il y a un moment comme celui-ci c'est tellement insaisissable que le simple fait d'en parler peut tout gâcher.

J.-P. DENIzON : Comment un spectateur peut communiquer l'émotion qu'il a ressenti avec CARMEN à quelqu'un d'autre qui n'a pas vu le spectacle? On lui expliquera CARMEN, mais il ne pourra pas le ressentir car il ne l'aura pas vu. Il n'aura pas été là à ce moment précis et lorsque c'est passé c'est fini. On en garde le souvenir mais uniquement pour soi-même. Ou bien encore ce spectateur dit à quelqu'un d'autre : «il faut absolument voir la pièce ». Celui-ci ira voir la pièce mais il y trouvera une autre émotion et ce ne sera jamais le même spectacle.

0. zонаR : Toutefois, lorsque je parle avec vous je ressens une certaine harmonie même si je ne sais pas d'où elle vient? Vous êtes en accord sur beaucoup de choses. Je ne retrouve pas chez vous ce que l'on rencontre habituellement dans les théâtres traditionnels, des acteurs qui ne sont jamais d'accord avec ce qu'exprime un autre comédien. II y a tout de suite contradiction.

A. MARATRA : Sur cela nous sommes d'accord, ce qui ne veut pas dire que nous soyons d'accord sur tout. Par exemple lorsqu'un sentiment est juste et qu'il passe bien il n'y a aucune contradiction. Lorsque c'est bien, nous sommes suffisamment sensibles pour l'admettre et nous sommes tous en accord. Néanmoins, il y a de nombreux domaines avec lesquels nous ne faisons pas l'unité, sans cela nous ne serions pas un groupe et il n'y aurait aucune possibilité de travailler ensemble.

J.-P. DENIzon : Disons que nous sommes d'accord sur ce que nous recherchons, sur le fond et notre façon d'être et d'agir.

O. zoнar : Comment réussissez-vous à être en accord sur la recherche et sur le fond, car dans le théâtre habituellement on trouve beaucoup d'attitudes artificielles, énormément de maniérismes, toutes sortes de clichés.

J.-P. DENIzoN : Cela tient peut-être à la manière de Peter BROOK de réunir les gens autour de lui.

O. ZOHAR : Qu'y a-t-il chez lui de si particulier?

J.-P. DENIZON : Je crois que cela réside dans son mode de recrutement. Il n'engage pas comme la plupart des metteurs en scène en pensant que tel acteur interprétera bien tel ou tel personnage. Je crois qu'il engage sur discussion et je pense qu'il a engagé Alain ainsi. Donc on voit à peu près où l'on va. Il est évident qu'en cinq minutes lorsque l'on parle avec les gens de théâtre et que l'on connaît bien ce métier, ce "métier » je n'aime pas ce terme enfin disons-le quand même, il est évident qu'en cinq minutes on voit tout de suite ce que les gens recherchent ou ne veulent pas faire. Cela se perçoit très rapidement.

A. MARATRA : Ce que l'on peut donner se voit immédiatement. Dans la troupe chacun de nous donne quelque chose de totalement différent. Si nous étions deux par exemple à vouloir jouer le même rôle, si j'étais identique à Jean-Paul et si mon caractère ne différait pas du sien, si j'avais le même aspect, là nous commencerions à être en désaccord. Nous serions en concurrence car nous aurions les mêmes fonctions. Un proverbe dit : « il n'y jamais deux tigres dans une même forêt ». Ainsi à titre d'exemple, l'une de nos six CARMEN la chanteuse israélienne Zehava GAL. Combien il est intéressant de remarquer la différence de sa personnalité et de son jeu. Il y a six 
Carmen, six autres actrices qui jouent pourtant le même caractère et il faut les voir jouer, le jour et la nuit, à midi, à trois heures de l'après-midi, toujours différentes c'est inimaginable. Et notre chanteuse autrichienne Eva SAUROVA, c'est incroyable de voir ce que cette chanteuse d'opéra est capable de réaliser tout simplement parce qu'on la laisse faire, subitement c'est elle qui donne tout. Même si les chanteuses discutent entre elles sur le caractère de CARMEN, car on est toujours tenté de dire de manière impérative : «le caractère de CARMEN c'est cela », elles ne sont pas d'accord sur l'essence, il n'y a pas qu'une seule façon de jouer CARMEN. On dit oui pour une idée, par exemple CARMEN est une fille complètement nerveuse mais cela non plus n'est pas vrai. EVA, joue CARMEN d'une toute autre manière et complètement crédible. Avant les représentations nous discutions sur la manière de jouer CARMEN. En poursuivant le travail par la suite, nous avons constaté que lorsque le spectacle de CARMEN commençait, apparaissait une CARMEN avec une certaine unité, un escalier... et l'histoire passait. Cela dépasse l'Être, la personne qui joue, le spectacle même. L'histoire qui est racontée dépasse tout... encore une question?

0. zoHAR : Lors de cette rencontre avec Peter au moment de votre engagement, y a-t-il eu une conversation importante?

J.-P. Denizon : Non, c'est une conversation normale. Toutefois, je peux vous dire qu'en discutant avec lui on voit très vite où l'on peut aller. Lorsque l'on rencontre une personne, en lui parlant on voit tout de suite ce qui émane d'elle, cela se sent. Il passe énormément de choses forcément. On a envie de la connaître ou de la rejeter. Il peut aussi se produire qu'il ne se passe rien. Lors d'un entretien beaucoup de choses se ressentent. En plus, nous sommes là pour parler d'un thème unique : le théâtre. Lorsque l'on est convoqué par un metteur en scène on ne va pas lui parler du marché... En parlant on arrive très vite à l'essentiel des choses, en deux ou trois questions très banales et très simples.

0. zонав : Peter ввоок a néanmoins l'art de poser des questions essentielles !

J.-P. DENIZON : Oui.

O. zoнAR : J'ai remarqué que dans CARMEN vous ne chantez pas du tout.

J.-P. DENIzon : Non, nous... ou plutôt en ce qui me concerne, je ne suis pas chanteur.

O. zoHAR : Pourtant dans TA-DA-DA vous chantiez tous les deux merveilleusement bien. J'ai pu constater aussi vos qualités d'acteurs qui passaient en chantant. Je voulais vous poser une dernière question. Peter a dit qu'il sera un jour nécessaire que ces murs chantent... Estce que vous pensez que les murs ont vraiment chanté lors des représentations dont nous avons parlées?

J.-P. DENIzon : Oui, cela s'est produit plus d'une fois, mais cela n'arrive pas tous les jours. 
0. zOHAR : Merci.

\section{NOTES}

1. 